\title{
Characterisation of guinea pig precision-cut lung slices: comparison with human tissues
}

\author{
A.R. Ressmeyer*, A.K. Larsson\#, E. Vollmer*, S.E. Dahlèn\#, S. Uhlig*, and C. Martin*
}

ABSTRACT: Precision-cut lung slices (PCLS) allow comparison of the airway responses of different species under identical experimental conditions. The aim of this study was to establish and characterise PCLS from guinea pigs (GPs) and to compare them with human PCLS.

GP PCLS were prepared according to previously published procedures with the exception that the agarose solution and the initial incubation medium contained isoproterenol to avoid post mortem airway contraction.

The median effective concentrations (EC50, expressed as $\mathrm{nM}$ ) for agonist-induced bronchoconstriction in GP and human PCLS, respectively, were: leukotriene $D_{4}(1.8,5.0)$; thromboxane $(16,1.3)$; serotonin $(69$, unresponsive); histamine $(217,2,170)$; and methacholine $(231,234)$. Allergen-induced bronchoconstriction of passively sensitised PCLS was attenuated by histamine or thromboxane-prostanoid receptor antagonists and was almost completely prevented by their combination with leukotriene receptor antagonists. Airways pre-contracted with methacholine were relaxed by the $\beta$-agonist salbutamol or the phosphodiesterase inhibitor 3-isobutyl-1methylxanthine. Simultaneous studies of airways and vessels are possible with, for example, EC50 values for endothelin-1 of $37 \mathrm{nM}$ (pulmonary arteries), $10 \mathrm{nM}$ (pulmonary veins) and $9.6 \mathrm{nM}$ (airway).

When compared with previous findings in rat and mouse, these data show that guinea pig lungs are a more appropriate model for human airway pharmacology than lungs from rats or mice.

KEYWORDS: Airway smooth muscle, eicosanoids, live dead staining, lung explants, ovalbumin, 2photon microscopy

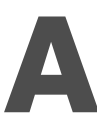

irway pharmacology varies strikingly between species. For instance, rodent airways do not respond or respond only weakly to leukotrienes [1], mediators that readily cause bronchoconstriction in humans [2] and guinea pigs (GPs) [3, 4]. GPs, which are not rodents [5], are widely used in pulmonary pharmacology, because their airways' responsiveness to mediators and drugs is thought to resemble human airways more closely than those of mice or rats [6,7]. However, most studies have focused either on in vivo experiments or on large airway preparations, both of which are difficult to translate to studies with humans, making direct interspecies comparisons difficult. In addition, the significance of peripheral airways for the pathology of asthma has only recently been appreciated [8-11].

A recently developed alternative to the classical pharmacological models is the precision-cut lung slice (PCLS) model. This was originally devised for toxicological studies [12-14], but the utilisation of PCLS from rats, mice and humans for studies of peripheral airway pharmacology has now been established $[1,9,15]$. The PCLS model has many advantages. First, since slices are viewed under the microscope, it is possible to study airways and vessels of different sizes and anatomical locations. For instance, rat and human PCLS small airways respond more strongly than larger airways to methacholine (Mch) [15], serotonin [8], thromboxane [16] and allergen [8, 9], but not to endothelin-1 [17]. Secondly, the slices are usually studied in 24-well plates requiring only $500-1,000 \mu \mathrm{L}$ bathing solution, facilitating experiments using expensive or rare drugs. Up to 30 slices can be prepared from one lung, which not only limits the number of experimental animals used, but can also help to reduce experimental error as it allows statistical blocking. Thirdly, the slices are available for experimentation for $\geqslant 3$ days, making it possible to study the long-term effects of cytokines or hormones, or the in vitro effects of molecular strategies such as interference RNA. Finally, PCLS provide the opportunity to examine the same experimental model in different species. This seems of particular importance at a time when differences between different asthma
AFFILIATIONS

${ }^{\star}$ Research Center Borstel, Borstel, and,

"Institute of Pharmacology and Toxicology, RWTH Aachen, Aachen, Germany.

\#Unit for Experimental Asthma and Allergy Research, Division of Physiology, National Institute of Environmental Medicine, Karolinska Institutet, Stockholm, Sweden.

\section{CORRESPONDENCE}

C. Martin

Division Pulmonary Pharmacology

Research Center Borstel

Leibniz Center for Medicine and

Biosciences

Parkallee 22

D-23845 Borstel

Germany

Fax: 494537188778

E-mail: cmartin@fz-borstel.de

Received:

January 122006

Accepted after revision:

May 222006

SUPPORT STATEMENT

A.R. Ressmeyer and this study were funded by the Deutsche Forschungsgemeinschaft GRK 288/ C7. S.E. Dahlèn is supported by the following Swedish foundations: Medical Research Council, Heart Lung Foundation, and Foundation for Allergy and Health Care Research (Vårdal). The work of A.K. Larsson was supported by the European Respiratory Society Fellowship Grants 521. 
models and also between asthma models and human asthma are increasingly recognised as a major impediment to drug development $[18,19]$.

Given the reported similarities between GP and human airway responses, PCLS from GP would be a valuable tool in airway pharmacology, but their preparation and properties have not been reported so far. SHI and co-workers [20, 21] have used razor-cut lung explants (diameter $1 \mathrm{~mm}$ ) and reported vascular responses to histamine and serotonin. The absence of airway data in these studies presumably relates to the practical problems of obtaining unobstructed airways in GPs. As previously reported in the isolated perfused GP lung [22], post mortem airway constriction invariably occurs unless specific measures are taken. Here, the current authors describe a method for preparation of PCLS from GPs, show that these PCLS stay viable for at least 3 days and demonstrate that the airways and vessels respond adequately to various mediators and to allergen once the post mortem constriction has been eliminated by specific precautions during the preparation procedure. These findings allow direct comparisons of airway responses of GPs to those of rats or humans in the same model.

\section{MATERIALS AND METHODS}

\section{Animals and material}

Female Dunken Hartley GPs $(350 \pm 30 \mathrm{~g})$ were obtained from Charles River (Sulzfeld, Germany). All agonists and antagonists were purchased from Sigma-Aldrich (Steinheim, Germany), except leukotriene (LT) $\mathrm{D}_{4}$ from Biomol (Hamburg, Germany), endothelin-1 from Bachem (Weil, Germany), and U46619 from Cayman Chemicals (Ann Arbor, MI, USA). Animal experiments and access to human lung material were approved by the local ethics committee.

\section{Precision-cut lung slices}

GP PCLS were prepared as previously described for other species (i.e. rat, mouse) $[1,15]$ with the following modifications. After injection of pentobarbital (Narcoren; Pharmazeutische Handelsgesellschaft $\mathrm{mbH}$, Garbsen, Germany; $\left.95 \mathrm{mg} \cdot \mathrm{kg}^{-1}\right)$ the trachea was cannulated and the animals exsanguinated by cutting the vena cava inferior. Through the cannula, the lung was filled with a low melting-point agarose solution $(0.75 \%$, final concentration) containing isoproterenol $(1 \mu \mathrm{M})$. In order to solidify the agarose and harden them for cutting, the lungs were placed on ice for $10 \mathrm{~min}$. The lobes were separated and tissue cores prepared with a rotating sharpened metal tube (diameter $8 \mathrm{~mm}$ ). These cores were cut into 220- $\mu$ m-thick slices with a Krumdieck tissue slicer (Alabama Research and Development, Munford, AL, USA). Human PCLS were prepared as previously described [9].

\section{Culture medium}

Tissue slices were incubated at $37^{\circ} \mathrm{C}$ in a humid atmosphere in minimal essential medium ( $\mathrm{pH} 7.2)$ composed of $\mathrm{CaCl}_{2}$ (1.8 mM), $\mathrm{MgSO}_{4}(0.8 \mathrm{mM}), \mathrm{KCl}(5.4 \mathrm{mM}), \mathrm{NaCl}(116.4 \mathrm{mM})$, glucose $(16.7 \mathrm{mM}), \mathrm{NaHCO}_{3}(26.1 \mathrm{mM})$, Hepes (25.17 mM), sodium pyruvate, amino acids, vitamins and glutamine [15]. Isoproterenol $(1 \mu \mathrm{M})$ was added initially and for up to the first $3 \mathrm{~h}$ of washings. The medium was changed every $30 \mathrm{~min}$ during the first $2 \mathrm{~h}$ followed by a change every $1 \mathrm{~h}$ for the next $2 \mathrm{~h}$, in order to remove the agarose and cell debris from the tissue. Subsequently, medium was further supplemented with penicillin and streptomycin and changed every $24 \mathrm{~h}$.

\section{Assessment of viability using lactate dehydrogenase}

The viability of the GP slices was assessed by measuring the relative amount of lactate dehydrogenase (LDH) released from the slices into the incubation medium, as previously described [15]. Three slices per well were placed into a 24 -well plate and covered with $1 \mathrm{~mL}$ incubation medium. At the indicated timepoints, slices were lysed in $1 \mathrm{~mL} 0.2 \%$ Triton X-100 solution and homogenised (Polytron; Kinematica AG, Littau, Switzerland). Cells and supernatant were analysed using a commercially available LDH assay (Dimension pan; Dade Behring, Schwalbach, Germany). The viability of the slices was expressed as the ratio of $\mathrm{LDH}$ in the supernatant to the total $\mathrm{LDH}$ (sum of LDH in slices and the supernatatant).

\section{Assessment of viability using two-photon microscopy}

To visualise the viability of the GP PCLS, two-photon microscopy was used in combination with the LIVE/DEAD $\mathbb{R}$ viability/cytotoxicity assay kit (Molecular Probes, Eugene, OR, USA). PCLS were incubated with $5 \mu \mathrm{M}$ acetomethylester of calcein (calcein AM; live staining) and $10 \mu \mathrm{M}$ ethidium homodimer (EthD; dead staining) for $40 \mathrm{~min}$ and washed to remove external dye. The fluorescent dyes were excited at $800 \mathrm{~nm}$ with a Ti:Sa femtosecond laser (Coherent, Dieburg, Germany). The laser beam was split into 64 individual beams (Trim Scope; LaVision BioTec, Bielefeld, Germany) that simultaneously excited and scanned the object on the microscope. Images were acquired using a digital camera (Imager QE; LaVision, Bielefeld, Germany). Emissions of calcein AM (emission filter 500/50 nm) for the cytoplasm (live staining, green) and of EthD (emission filter 625/50 nm) for the staining of nuclei (dead staining, blue) were recorded separately. Overlay images of both dyes were shown. Slices were analysed 24,48 and $72 \mathrm{~h}$ after preparation. To visualise the total amount of dead cells, some PCLS were treated with 1\% Triton X-100 for $20 \mathrm{~min}$ prior to incubation with dyes.

\section{Measurement and imaging}

The airways and vessels were imaged and digitised using a digital video camera as previously described [8]. Airway or vessel area before addition of the mediators was defined as $100 \%$. Bronchoconstriction or vasoconstriction was expressed as airway/vessel area as the percentage of the initial area. For the measurements, slices with comparable airway size were selected. These slices were put on 24-well plates and fixed with a nylon thread attached to a platinum wire, in order to avoid movement of the slice during measurement. The slices were kept covered with $1 \mathrm{~mL}$ incubation medium and maintained at $\sim 37^{\circ} \mathrm{C}$.

The 24-well plate was positioned on the stage of an inverted microscope. Images were recorded by analogue (JAI 2040; JAI Pulnix, Alzenau, Germany) or digital camera (IRB640; Visitron Systems, Munich, Germany). A control image was taken before addition of the mediator, and frames were recorded every $30 \mathrm{~s}$ for 5,10 or 20 min depending on the study. In order to control maximal dilation of airways, some PCLS were incubated with salbutamol $(10 \mu \mathrm{M})$. Less than $10 \%$ of the airways were 
pre-contracted. Those which were pre-contracted showed a maximal dilation to $113 \%(100=$ baseline $)$.

\section{Passive sensitisation}

GP PCLS were treated and incubated overnight with $1 \%$ serum from GPs that had been actively sensitised with ovalbumin using standard protocols [7]. The next day, slices were transferred onto a 24-well plate with fresh medium and put under the microscope. A control image was taken before the allergen was added.

\section{Mediator-induced bronchoconstriction}

To compare agonists involved in the allergen-induced bronchoconstriction in GP and human PCLS, cumulative concentrationresponse curves for histamine $(0.001-100 \mu \mathrm{M})$, serotonin (0.0001-10 $\mu \mathrm{M}), \mathrm{LTD}_{4}(0.01-1,000 \mathrm{nM})$, thromboxane-prostanoid (TP) agonist U46619 (0.01-1,000 nM) and Mch (0.001-10 $\mu \mathrm{M})$ were established.

\section{Pharmacological intervention studies}

To determine the mediators involved in allergen-induced bronchoconstriction, PCLS were pre-incubated for $\geqslant 10 \mathrm{~min}$ with the cysteinyl leukotriene-1 $\left(\right.$ cysLT $\left._{1}\right)$ receptor antagonist montelukast $(10 \mu \mathrm{M})$, the TP receptor antagonist SQ29548 $(10 \mu \mathrm{M})$, or the histamine- $1\left(\mathrm{H}_{1}\right)$ receptor antagonist triprolidine $(5 \mu \mathrm{M})$ alone or in combination before addition of a single dose of ovalbumin $\left(100 \mathrm{ng} \cdot \mathrm{mL}^{-1}\right)$.

\section{Airway relaxation}

For the relaxation experiments, PCLS were contracted with Mch $(316 \mathrm{nM})$ to $10-20 \%$ of their initial airway area. Subsequently, increasing concentrations of the $\beta$-agonist salbutamol (10 nM-10 $\mu \mathrm{M})$, the unspecific phosphodiesterase (PDE) inhibitor 3-isobutyl-1-methylxanthine (IBMX; $10 \mathrm{nM}-$ $10 \mu \mathrm{M})$ and the combination of both were added in the presence of Mch.

\section{Statistics}

Percentage data for bronchoconstriction were transformed by the arcsin-transformation prior to analysis. The data were analysed by two-sided paired t-tests or three-way factorial mixed model analysis as indicated. The p-values were corrected for multiple comparisons according to the falsediscovery rate procedure. The concentration-response curves were analysed as sigmoidal dose-response curves.

\section{RESULTS}

Applied to GP lungs, standard preparation protocols established for rat or mouse slices always produced PCLS in which the airways closed almost completely within the first $10 \mathrm{~min}$ after preparation, whereupon they did not reopen for hours. Attempts to prevent this post mortem bronchoconstriction by incubation and prolonged washing in the presence of the muscarinic-receptor antagonist atropine, SQ29548, montelukast or triprolidine failed, as did addition of a cocktail of all inhibitors (data not shown). In contrast, inclusion of the $\beta$ agonist isoproterenol $(1 \mu \mathrm{M})$ in the agarose medium as well as in the medium used for cutting and washing completely prevented the post mortem constrictions. This procedure allowed the current authors to obtain up to 30 slices with open airways from a single GP lung. After the first $3 \mathrm{~h}$ of washings, isoproterenol exposure was discontinued, but the airways nevertheless remained open and responsive. The use of the selective $\beta_{2}$-agonist salbutamol was also examined, but the drug was not suitable because of its longer duration of action in this preparation.

The viability of GP PCLS was determined by measurement of the release of LDH into the medium (fig. 1) and by two-photon microscopy over a period of 3 days (fig. 2). LDH leakage remained $<6 \%$ during this time when the medium was changed every $24 \mathrm{~h}$, and was $17 \%$ when the medium was unchanged for $72 \mathrm{~h}$. Microscopic determination of viable cells showed $<10 \%$ dead cells in the second or third cell layer (fig. 2a-d; all nuclei are visible in fig. 2d). The first cell layer was partly disrupted by the slicing procedure (data not shown). The number of dead cells remained stable over a period of $72 \mathrm{~h}$, indicating again that a daily medium change is effective in keeping slices viable for at least 3 days.

The current authors examined how the airways from GP and human PCLS responded to mediators relevant to asthma (table 1; fig. 3). In GPs, the biogenic amines serotonin and histamine caused bronchoconstriction with median effective concentrations (EC50) of $69 \mathrm{nM}$ and $217 \mathrm{nM}$, respectively, while in humans, histamine (EC50 $2.7 \mu \mathrm{M})$ was effective, but not serotonin. In GP PCLS, the histamine-induced airway constriction was unaltered by the TP-receptor antagonist SQ29548 $(10 \mu \mathrm{M})$, suggesting a predominantly direct effect of histamine in this preparation (data not shown). Mch, a stable acetylcholine derivative, contracted airways with nearly identical EC50 values $(\sim 230 \mathrm{nM})$ in GP and humans. The most potent bronchoconstrictors were eicosanoids: the stable TP receptor agonist U46619 (GP: EC50 $16 \mathrm{nM}$; human: EC50 $1.3 \mathrm{nM}$ ) and LTD $_{4}$ (GP: EC50 1.8 nM; human: EC50 5.0 nM). In GP PCLS, prostaglandin $\mathrm{D}_{2}$ was somewhat less potent, with an EC50 of $175 \mathrm{nM}$ (data not shown).

To assess whether an allergen-induced bronchoconstriction could be evoked in this new model, GP PCLS were passively

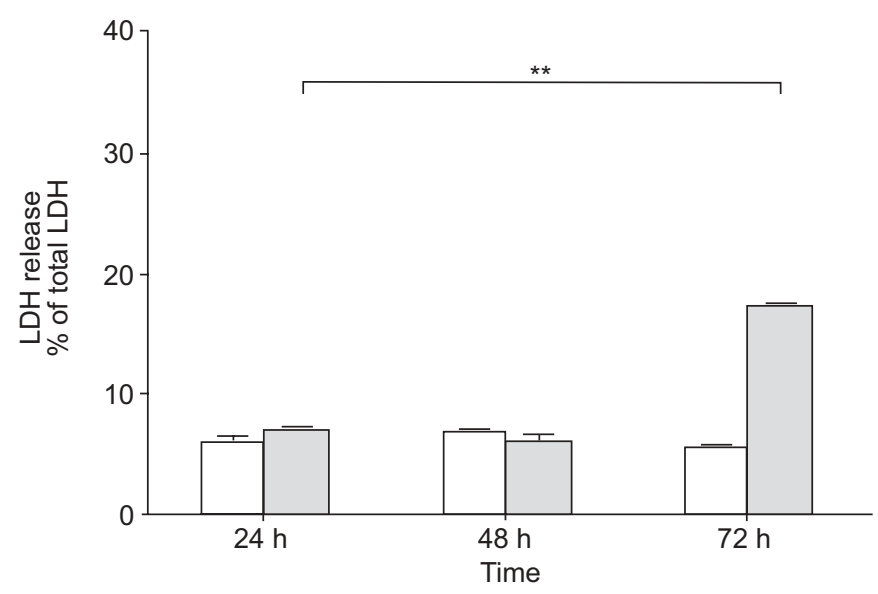

FIGURE 1. Lactate dehydrogenase (LDH) release in precision-cut lung slices (PCLS). PCLS were cultured for $72 \mathrm{~h}$ in minimum essential medium with $(\square ; n=4)$ or without $(\square ; n=4)$ medium change every $24 h .{ }^{*}: p<0.01$, two-sided t-test. Data are presented as mean \pm SE as percentage of initial area. 

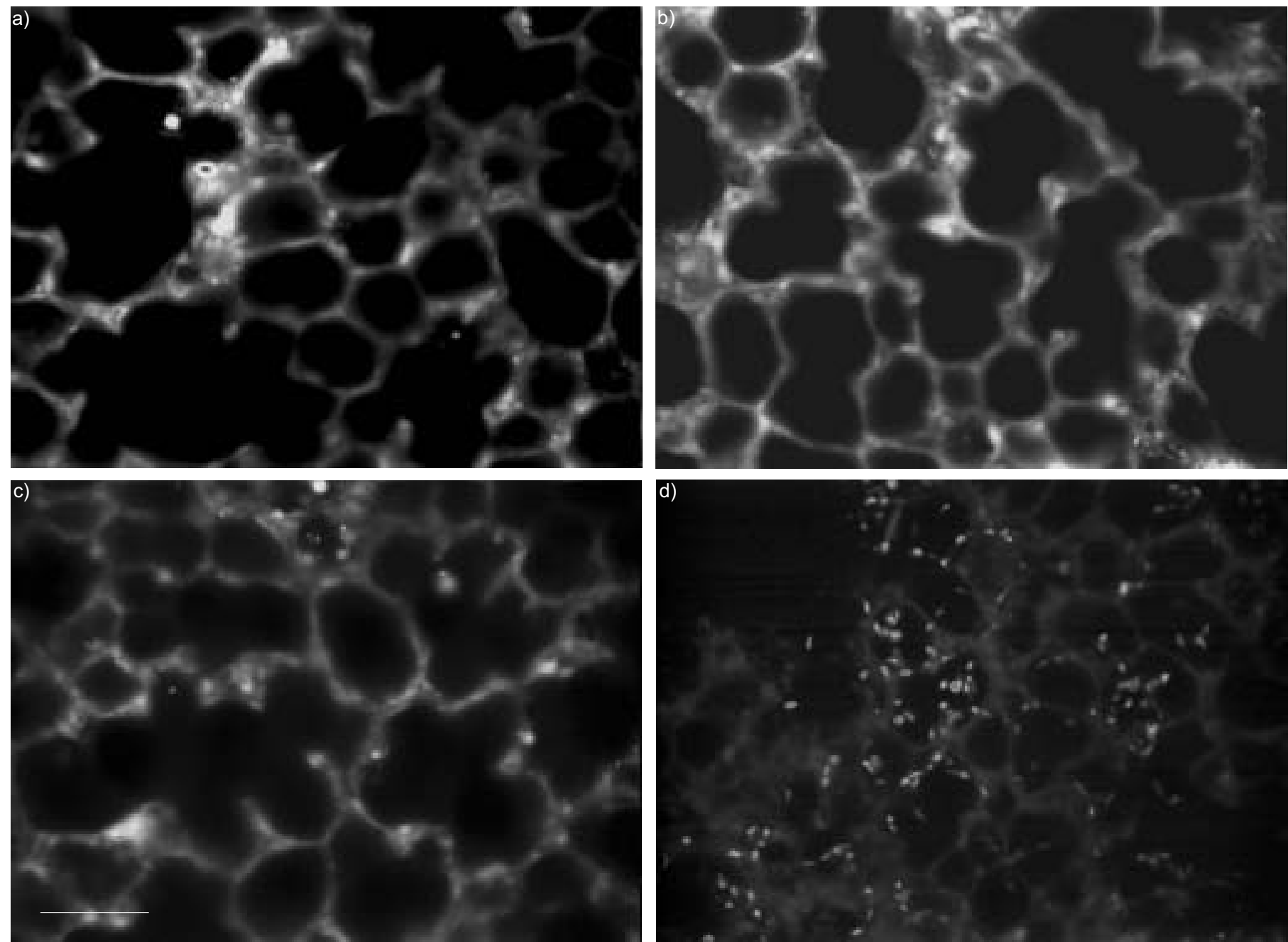

FIGURE 2. Two-photon microscopic image of guinea pig precision-cut lung slices (PCLS) stained with calcein acetomethylester (AM) and ethidium homodimer (EthD-1) A 220- $\mu \mathrm{m}$-thick PCLS was loaded with $5 \mu \mathrm{M}$ calcein AM/10 $\mu \mathrm{M}$ EthD-1 for $40 \mathrm{~min}$ and excited at $800 \mathrm{~nm}$ with a femtosecond laser. The images show overlay frames (cytoplasm and nuclei). Emission was selected by 500/50 and 625/50 filters for calcein AM and EthD-1, respectively. Viable PCLS at a) 24 h, b) 48 h, and c) 72 h. d) A PCLS pre-treated with $1 \%$ Triton $\mathrm{X}-100$ for 20 min followed by LIVE/DEAD staining. The percentages of nuclei in $\mathrm{a}, \mathrm{b}$ and $\mathrm{c}$ compared to $\mathrm{d}$ were $8.6,4.9$ and $5.5 \%$, respectively Scale bar $=80 \mu \mathrm{m}$.

sensitised overnight with serum from ovalbumin-sensitised GPs, and subsequently exposed to the allergen. Addition of ovalbumin to passively sensitised slices caused a concentration-dependent bronchoconstriction with an EC50 for ovalbumin of $3.8 \mathrm{ng} \cdot \mathrm{mL}^{-1}$ (fig. 4).
In order to analyse the mediators responsible for the allergeninduced bronchoconstriction in this new model, PCLS were pre-incubated with three different inhibitors: triprolidine $(5 \mu \mathrm{M})$, antagonist SQ29548 $(10 \mu \mathrm{M})$ and montelukast $(1 \mu \mathrm{M})$. These inhibitors were used alone and in all possible

TABLE 1 Median effective concentrations (EC50) for agonist-induced airway contractions in different in vitro models

\begin{tabular}{lcccc} 
Agent & PCLS GP pD50 & PCLS GP EC50 nM & PCLS human pD50 & PCLS human EC50 nM \\
\hline LTD $_{\mathbf{4}}$ & $8.7 \pm 0.10$ & 1.8 & $8.3 \pm 0.10$ & 5.0 \\
U46619 & $7.8 \pm 0.07$ & 16 & $8.8 \pm 0.21$ & 1.3 \\
Serotonin & $7.2 \pm 0.02$ & 69 & & \\
Histamine & $6.7 \pm 0.07$ & 217 & $5.6 \pm 0.22$ & 2710 \\
Methacholine & $6.6 \pm 0.10$ & 231 & $6.6 \pm 0.10$ & 234 \\
\hline
\end{tabular}

Data are presented as mean \pm SE. PCLS: precision-cut lung slice; GP: guinea pig; pD50: potency $\left(-\log \left(E_{5} 5\right)\right)$; $L_{T D}$ : leukotriene $D_{4}$ 


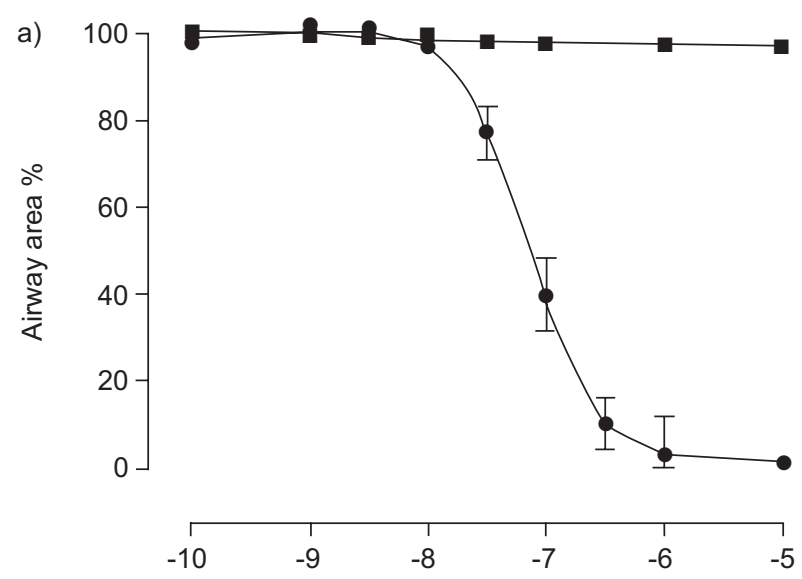

b)
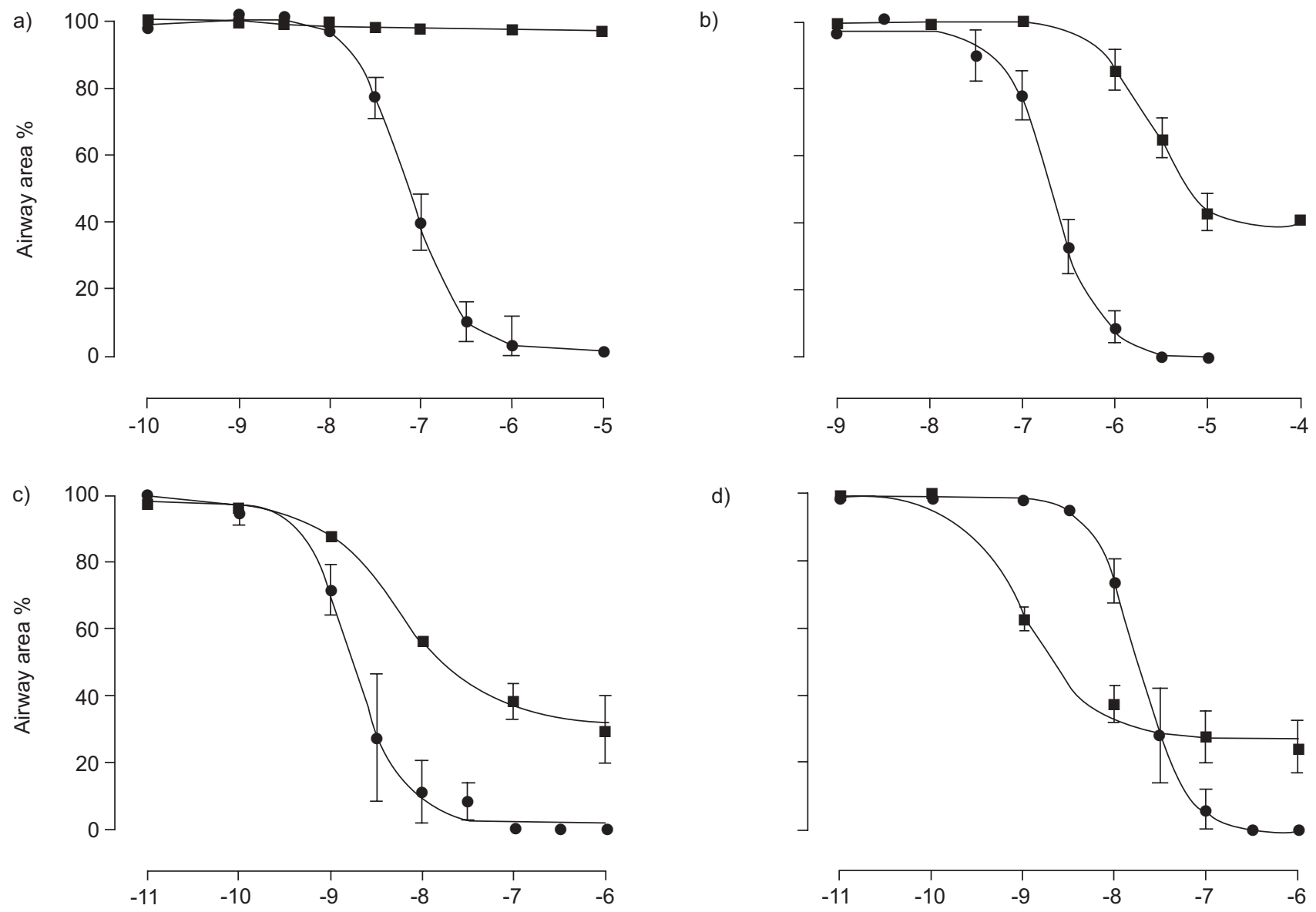

d)
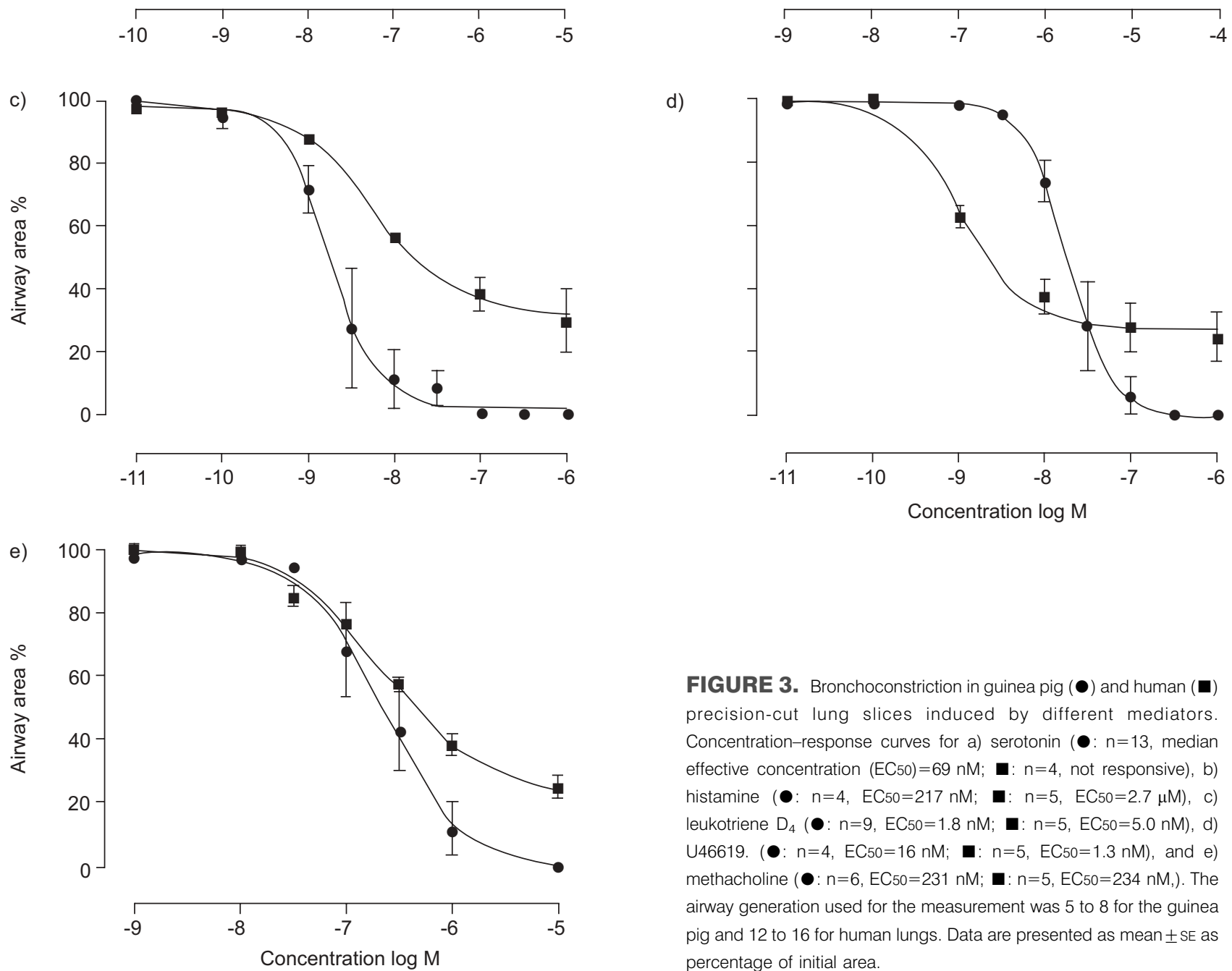

combinations, giving a full factorial design (fig. 4b). Pretreatment with triprolidine or SQ29548 attenuated the allergeninduced bronchoconstriction, and the effects of these two inhibitors were additive. Montelukast was without effect when given alone, but was effective in combination with triprolidine.

The study also examined two commonly used bronchodilators, the $\beta_{2}$-agonist salbutamol and the unspecific PDE inhibitor IBMX. After pre-contracting airways with Mch to $10-20 \%$ of their initial airway area, salbutamol, IBMX or a combination of the two were added cumulatively. These interventions

produced a concentration-related reversal of Mch-induced contractions with significant effects of salbutamol above $100 \mathrm{nM}$ and IBMX at $10 \mu \mathrm{M}$ (fig. 5). The combination of both bronchodilators showed a synergistic effect and was effective above concentrations of $100 \mathrm{nM}$ of each drug (fig. 5c).

A particularly useful feature of PCLS is the possibility to measure both airway and vascular responses. This is illustrated in figure 6, which shows the response of an airway, a pulmonary artery and a pulmonary vein to endothelin-1. Pulmonary artery and vein can be distinguished by their precision-cut lung slices induced by different mediators. Concentration-response curves for a) serotonin $(\boldsymbol{\bullet}: \mathrm{n}=13$, median effective concentration (EC50) $=69 \mathrm{nM} ; \mathbf{\square}: \mathrm{n}=4$, not responsive), b) histamine $(\bullet: \mathrm{n}=4, \mathrm{EC} 50=217 \mathrm{nM} ; \mathbf{\square}: \mathrm{n}=5, \mathrm{EC} 50=2.7 \mu \mathrm{M}), \mathrm{c})$ leukotriene $\left.\mathrm{D}_{4}(\boldsymbol{\bullet}: \mathrm{n}=9, \mathrm{EC} 50=1.8 \mathrm{nM} ; \mathbf{\square}: \mathrm{n}=5, \mathrm{EC} 50=5.0 \mathrm{nM}), \mathrm{d}\right)$ 46619. ( $\left.: \mathrm{n}=4, \mathrm{EC}_{50}=16 \mathrm{nM} ; \boldsymbol{\square}: \mathrm{n}=5, \mathrm{EC}_{50}=1.3 \mathrm{nM}\right)$, and e) 列 pig and 12 to 16 for human lungs. Data are presented as mean \pm SE as percentage of initial area. 

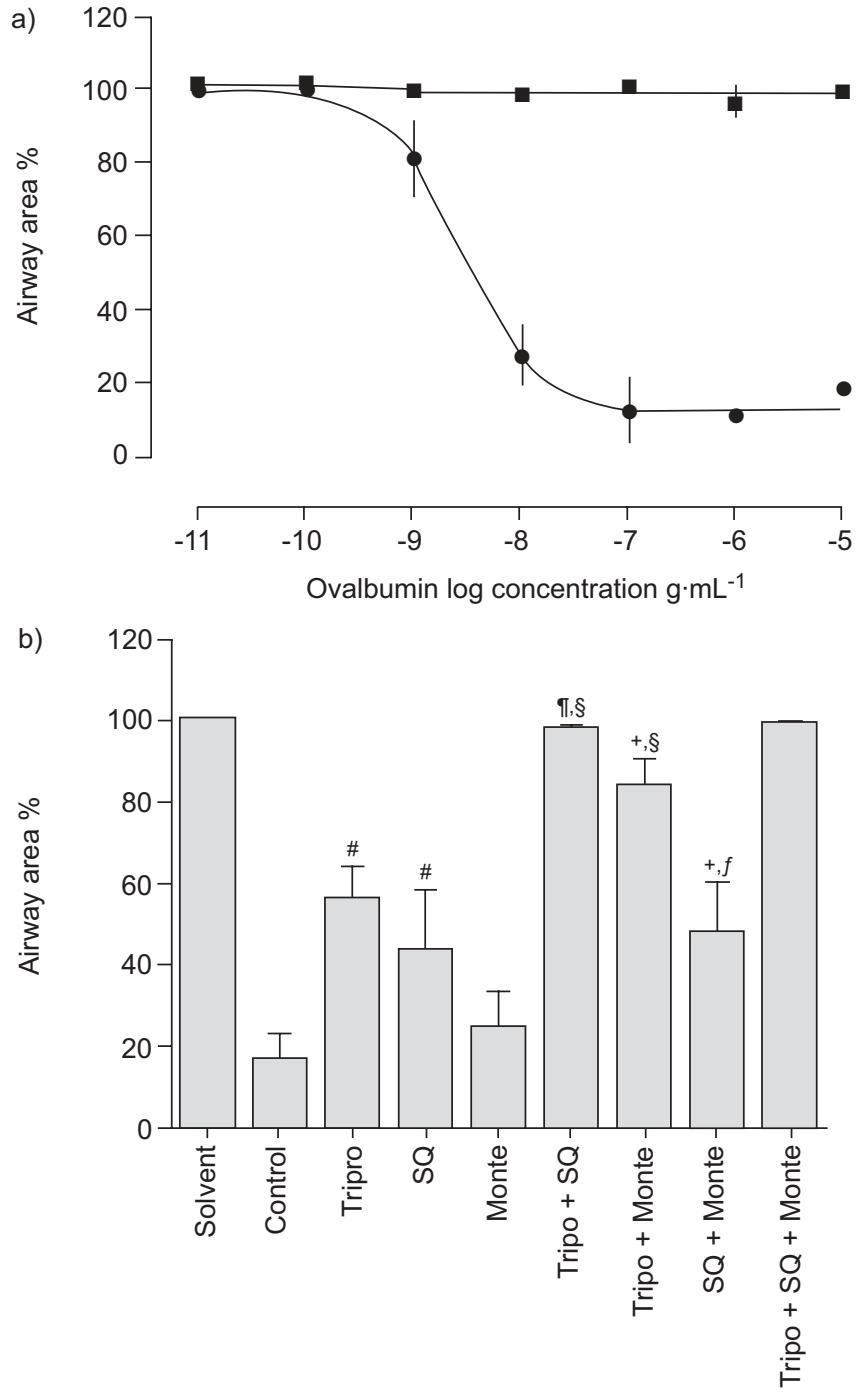

FIGURE 4. Ovalbumin-induced bronchoconstriction in sensitised guinea pig (GP) precision-cut lung slices (PCLS). a) Cumulative concentration-response curve in passive sensitised $(\bullet)$ and nonsensitised ( $\bullet$ PCLS with a median effective concentration of $3.8 \mathrm{ng} \cdot \mathrm{mL}^{-1}$. Similar results were obtained if each ovalbumin concentration was tested in a separate slice (data not shown). b) Pharmacological inhibition of allergen-induced bronchoconstriction. The effects of receptor antagonists for the histamine-1 receptor ( $5 \mu \mathrm{M}$ triprolidine; Tripro), the thromboxane-prostanoid receptor (10 $\mu \mathrm{M} \mathrm{SQ29845;} \mathrm{SQ)} \mathrm{and} \mathrm{the} \mathrm{cysteinyl}$ leukotriene-1 receptor (10 $\mu \mathrm{M}$ montelukast; Monte) alone or in combination are shown. The data were analysed by mixed model analysis with inhibitors as fixed factors and subjects (GPs) as random factor; subsequently individual contrasts were calculated and corrected by the false discovery rate procedure. ${ }^{*}: p<0.01$ versus solvent control; ${ }^{\text {": }} p<0.01$ versus SQ; ${ }^{+}: p<0.01$ versus Monte; ${ }^{\text {s: }} p<0.01$ versus Tripro; ${ }^{f}: p<0.01$ versus all three inhibitors. Data are presented as mean $\pm \mathrm{SE}$. The number of independent experiments was as follows: control $n=12$; Tripro $n=8$; $\mathrm{SQ} \mathrm{n}=8$; Monte $\mathrm{n}=5$; Tripro $+\mathrm{SQ} \mathrm{n}=4$; Tripro + Monte $\mathrm{n}=5 ; \mathrm{SQ}+$ Monte $\mathrm{n}=5$; Tripro + SQ + Monte $\mathrm{n}=3$.

position relative to the airway and by the amount of smooth muscle. EC50 values for the endothelin-1-induced contraction of airways, pulmonary artery and pulmonary vein were 9.6, 37, and $10 \mathrm{nM}$, respectively. As previously observed in slices also from other species [23] as well as in perfused lung models [24], there was some perivascular oedema around the pulmonary artery.

\section{DISCUSSION}

For pharmacological and mechanistic studies relevant to human disease, differences in airway pharmacology between rodent models and human lungs remain an important problem. As the preparation of PCLS is essentially the same in all species, this model provides an excellent way to compare the airway pharmacologies of different species. PCLS allow investigation of single airways and vessels under cell culture conditions, and have already been used to elucidate important mechanisms of airway contraction in rat, mouse and human peripheral lungs $[1,8,15,16]$. The results of the current study show that PCLS from GPs correspond to human airways fairly well in terms of both mediator and allergic airway responses, and are therefore more suitable than rodent PCLS for studies relevant to human airway pharmacology.

The present study describes for the first time the preparation and properties of GP PCLS. It is difficult to produce highquality slices from GPs because of post mortem bronchoconstriction that has been documented radiographically [25] and in isolated lungs [26]. This constriction has been attributed to the release of substance $P$ from sensory nerves (relaxing vessels at the same time), but other mediators or direct effects of changing microenvironment $\left(\mathrm{pH}, \mathrm{CO}_{2}\right)$ on airway muscle may also be involved [22]. Chronic treatment with capsaicin to empty substance $\mathrm{P}$ stores from sensory nerves, or in vivo pretreatment with morphine, prevented the post mortem bronchoconstriction [27]. However, such treatments are impractical for routine use, carry some ethical concerns as capsaicin treatment is painful, and may interfere with the purpose of the experiments. Here, the current authors report the successful elimination of the post mortem bronchoconstriction by the brief $(3 \mathrm{~h})$ initial inclusion of isoproterenol in all the media used for preparation of the PCLS. For the same reason, isoproterenol is also routinely used during the preparation of isolated perfused GP lungs [28]. Once the preparation of the PCLS is finished, isoproterenol treatment can be discontinued, whereupon airways and vessels respond normally to various stimuli, showing that the effect of isoproterenol is not sustained. This is supported by the expected bronchorelaxant effects observed with addition of salbutamol to pre-contracted, but not to untreated preparations.

Compared to classical models of studying airway functions in vitro, such as tracheal rings or parenchymal strips, PCLS offer many advantages such as economic use of expensive agents, longevity and, in particular, the possibility of studying airway and vascular responses simultaneously; PCLS even permit differentiation between pulmonary arteries and pulmonary veins in the same slice. This was demonstrated in the present study with endothelin-1, and in related studies using lung explants for histamine and serotonin [20, 21]. In all these studies, pulmonary veins responded more strongly than pulmonary arteries, corroborating many other findings in rat, sheep and human lungs [29].

GPs are frequently employed for studies in pulmonary pharmacology, because they are thought to possess a pharmacological profile $[6,7,30]$ similar to that of humans $[31,32]$. 

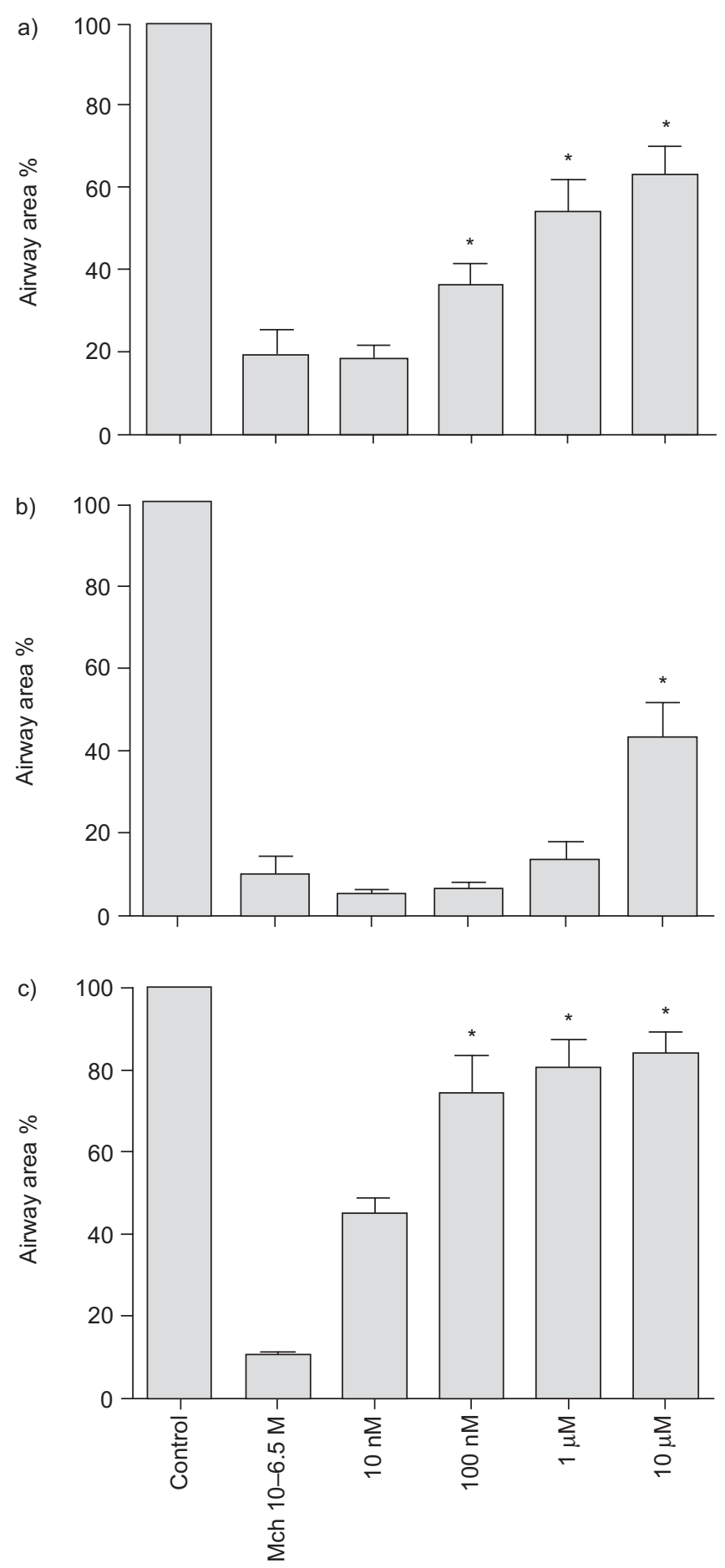

FIGURE 5. Relaxation of pre-contracted airways. Methacholine (MCh)-induced $(316 \mathrm{nM})$ airway contraction was reversed by a) salbutamol $(n=6)$, b) 3-isobutyl-1methylxanthine $(n=6)$, and $c)$ a combination of the two $(n=4)$. Data are presented as mean \pm SE as percentage of initial area. ${ }^{*}: p<0.05$ versus methacholine alone.

However, this assumption has never been thoroughly tested in a single laboratory using identical methodologies. Having at hand both GP and human PCLS, the current authors have been able to test this assumption and to define similarities and differences between the two species relevant to human asthma.
Comparison between GP and human airways was performed by examining the airways' responsiveness towards a variety of endogenous mediators (table 1) and, following passive sensitisation, towards allergen (table 2). All GP data are derived from the present study, whereas the allergen-induced bronchoconstriction data summarised in table 2 for human and rat PCLS have been taken from previous studies [8,9].

The EC50 values for human and GP bronchoconstriction are almost identical for $\mathrm{LTD}_{4}$ and Mch. Airways of both species responded to thromboxane and histamine, with human airways being more sensitive to thromboxane, and GP airways being more sensitive to histamine. The most significant difference was obtained for serotonin, which was quite effective in GP and completely ineffective in humans. In general, GP airways narrowed to a greater extent than human airways (table 1; fig. 3). The reason for this is not clear, but may, at least in part, relate to age differences in tissue (young healthy GPs versus middle-aged adults undergoing pulmectomy). Of note, the human PCLS were all from small airways (diameter $<2 \mathrm{~mm}$ ), and thus free of cartilage. Overall, the comparison between GP and human airways showed that GPs are not a perfect match but do nevertheless provide a reasonable approximation to humans. This is certainly true in comparison to mouse and rat airways, which do not respond or respond only weakly to leukotrienes and histamine [1, 8], mediators that play a role in human asthma [32].

With respect to the allergen-induced bronchoconstriction, the distance between humans and GPs on one side, and rats on the other side, is even more evident (table 2). In both GP and humans, both thromboxane and leukotrienes contribute to allergen-induced bronchoconstriction [9]. The major difference relates to histamine, which is clearly more important in GPs. However, none of these mediators plays a role in rats, where the allergen-induced bronchoconstriction is almost exclusively mediated by serotonin [8]. In the mouse, the mechanism of allergen-induced bronchoconstriction appears to be even more different, and apparently mast cell independent [33].

Thromboxane, leukotrienes and histamine (at least in GPs) are all potent bronchoconstrictors; therefore, it is to be expected that blockade of one mediator at a time will have only a small effect. This was indeed found to be the case, even though significant protection could be obtained with the antihistamine alone. The combination of the antihistamine with either TP or cysteinyl-leukotriene antagonists was particularly effective. In general, these results are in line with previous findings in other GP preparations such as perfused lungs [7], isolated tracheas [34] or parenchymal strips [30], which also show that histamine, leukotrienes and cyclo-oxygenase products contribute additively to allergen-induced bronchoconstriction. Those studies, however, employed cyclo-oxygenase inhibitors rather than TP receptor antagonists; in comparison, it appears that when given alone, cyclo-oxygenase inhibitors are more effective than TP receptor antagonists. This may indicate that other cyclo-oxygenase products such as prostaglandin $E_{2}$ (acting at contractile E-prostanoid ${ }_{1 / 3}$ receptors) and prostaglandin $\mathrm{F}_{2 \alpha}$ are also involved in allergen-induced bronchoconstriction. It is therefore possible that in those studies some cyclo-oxygenase products came from infiltrated eosinophils and other inflammatory cells. Nonetheless, all these 

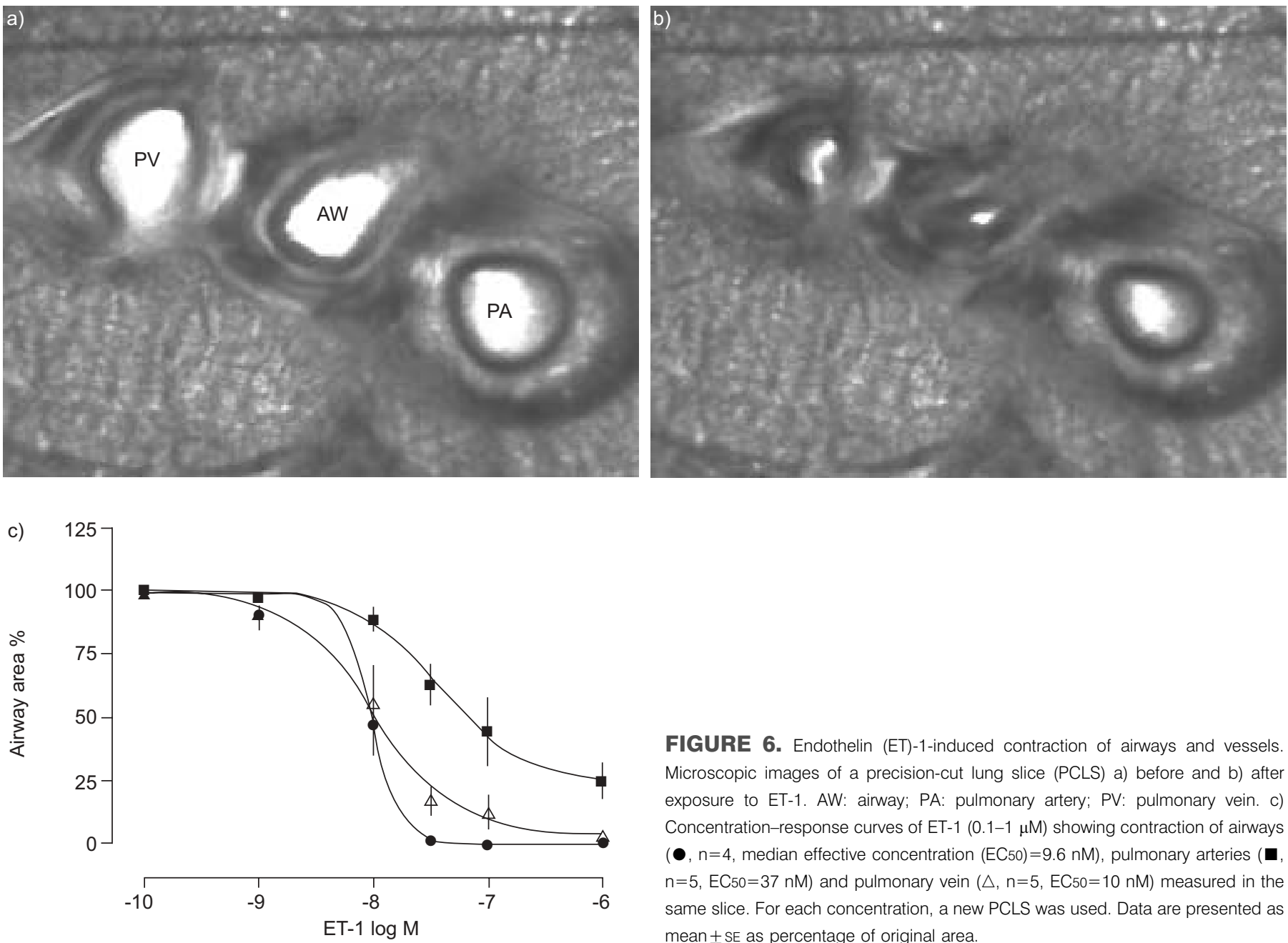

FIGURE 6. Endothelin (ET)-1-induced contraction of airways and vessels. Microscopic images of a precision-cut lung slice (PCLS) a) before and b) after exposure to ET-1. AW: airway; PA: pulmonary artery; PV: pulmonary vein. C) Concentration-response curves of ET-1 (0.1-1 $\mu \mathrm{M})$ showing contraction of airways $(\bullet, n=4$, median effective concentration $(E C 50)=9.6 \mathrm{nM})$, pulmonary arteries ( $n=5, E C 50=37 n M)$ and pulmonary vein $(\triangle, n=5, E C 50=10 n M)$ measured in the same slice. For each concentration, a new PCLS was used. Data are presented as mean \pm SE as percentage of original area.

experimental studies show that allergen-induced bronchoconstriction can only be significantly prevented if at least two of three bronchoactive mast cell mediators are blocked. In atopic subjects with asthma, antileukotriene drugs effectively inhibit both the early and late phases of allergen-induced bronchoconstriction $[7,30]$. This may seem to contrast with the current authors' inability to find a pronounced effect of the cysLT 1 receptor antagonist montelukast alone in the GP PCLS model. However, the airways examined in the bronchoprovocation

\begin{tabular}{|c|c|c|c|}
\hline TABLE 2 & $\begin{array}{l}\text { Mediators in } \\
\text { bronchocons } \\
\text { species }\end{array}$ & $\begin{array}{l}n \text { the allerg } \\
\text { of PCLS fr }\end{array}$ & $\begin{array}{l}\text { duced } \\
\text { ifferent }\end{array}$ \\
\hline & Rat [8] & Human [9] & Guinea pig \\
\hline Serotonin & $x$ & - & - \\
\hline Histamine & - & $(X)$ & $x$ \\
\hline Thromboxane & - & $x$ & $x$ \\
\hline Leukotrienes & - & $x$ & $x$ \\
\hline
\end{tabular}

studies in vivo are mainly central airways, whereas the PCLS and parenchymal strips focus on peripheral airways [7]. The relative contributions of the individual mediators may well differ along the airway tree and be related to differences in amounts of released mediators, airway geometry and expression of receptors. Again, when all three classes are blocked, there is complete protection [7, 9, 30, 32, 35].

In conclusion, this is the first study on precision-cut lung slices from guinea pig lungs. The slices are viable for at least 3 days and respond to mediators and allergens in a characteristic fashion that is similar to the response of human tissue. Although responses in the guinea pig airways displayed some differences compared with human tissue, the similarities appear greater than the differences. The current study shows that with respect to airway pharmacology, guinea pig precision-cut lung slices are the model that so far most closely resembles the human situation.

\section{ACKNOWLEDGEMENTS}

The authors gratefully acknowledge H. Magnussen and D. Brandscheid (Hospital Grosshansdorf, Grosshansdorf, Germany) for providing human lung material, and J. Sarau for technical assistance. 


\section{REFERENCES}

1 Held HD, Martin C, Uhlig S. Characterization of airway and vascular responses in murine lungs. $\mathrm{Br} J$ Pharmacol 1999; 126: 1191-1199.

2 Dahlèn SE, Hedqvist P, Hammarstrom S, Samuelsson B. Leukotrienes are potent constrictors of human bronchi. Nature 1980; 288: 484-486.

3 Drazen JM, Austen KF, Lewis RA, et al. Comparative airway and vascular activities of leukotrienes C-1 and D in vivo and in vitro. Proc Natl Acad Sci USA 1980; 77: 4354-4358.

4 Hedqvist P, Dahlèn SE, Gustafsson L, Hammarstrom S, Samuelsson B. Biological profile of leukotrienes C4 and D4. Acta Physiol Scand 1980; 110: 331-333.

5 D'Erchia AM, Gissi C, Pesole G, Saccone C, Arnason U. The guinea-pig is not a rodent. Nature 1996; 381: 597-600.

6 Muccitelli RM, Tucker SS, Hay DW, Torphy TJ, Wasserman MA. Is the guinea pig trachea a good in vitro model of human large and central airways? Comparison on leukotriene-, methacholine-, histamine- and antigeninduced contractions. J Pharmacol Exp Ther 1987; 243: 467-473.

7 Sundstrom E, Lastbom L, Ryrfeldt A, Dahlèn SE. Interactions among three classes of mediators explain antigen-induced bronchoconstriction in the isolated perfused and ventilated guinea pig lung. J Pharmacol Exp Ther 2003; 307: 408-418.

8 Wohlsen A, Uhlig S, Martin C. Immediate allergic response in small airways. Am J Respir Crit Care Med 2001; 163: 1462-1469.

9 Wohlsen A, Martin C, Vollmer E, et al. The early allergic response in small airways of human precision-cut lung slices. Eur Respir J 2003; 21: 1024-1032.

10 Wright JL. Diseases of the small airways. Lung 2001; 179: 375-396.

11 Shaw RJ, Djukanovic R, Tashkin DP, Millar AB, du Bois RM, Orr PA. The role of small airways in lung disease. Respir Med 2002; 96: 67-80.

12 Krumdieck CL, Dos Santos JE, Ho KJ. A new instrument for the rapid preparation of tissue slices. Anal Biochem 1980; 104: 118-123.

13 Fisher RL, Smith MS, Hasal SJ, Hasal KS, Gandolfi AJ, Brendel K. The use of human lung slices in toxicology. Hum Exp Toxicol 1994; 13: 466-471.

14 Stefaniak MS, Brendel K. Biochemical and histological characterization of agar-filled precision-cut rat lung slices in dynamic organ culture as an in vitro tool. In Vitro Toxicol 1992; 5: 7-19.

15 Martin C, Uhlig S, Ullrich V. Videomicroscopy of methacholine-induced contraction of individual airways in precision-cut lung slices. Eur Respir J 1996; 9: 2479-2487.

16 Martin C, Ullrich V, Uhlig S. Effects of the thromboxane receptor agonist U46619 and endothelin-1 on large and small airways. Eur Respir J 2000; 16: 316-323.

17 Martin C, Held HD, Uhlig S. Differential effects of the mixed ET(A)/ET(B)-receptor antagonist bosentan on endothelin-induced bronchoconstriction, vasoconstriction and prostacyclin release. Naunyn Schmiedebergs Arch Pharmacol 2000; 362: 128-136.
18 Coffman RL, Hessel EM. Nonhuman primate models of asthma. J Exp Med 2005; 201: 1875-1879.

19 Boyce JA, Austen KF. No audible wheezing: nuggets and conundrums from mouse asthma models. J Exp Med 2005; 201: 1869-1873.

20 Shi W, Eidelman DH, Michel RP. Differential relaxant responses of pulmonary arteries and veins in lung explants of guinea pigs. J Appl Physiol 1997; 83: 1476-1481.

21 Shi W, Wang CG, Dandurand RJ, Eidelman DH, Michel RP. Differential responses of pulmonary arteries and veins to histamine and 5-HT in lung explants of guinea-pigs. $\mathrm{Br} \mathrm{J}$ Pharmacol 1998; 123: 1525-1532.

22 Lai YL, Lamm WJ, Hildebrandt J. Factors affecting massive postmortem bronchoconstriction in guinea pig lungs. J Appl Physiol 1984; 57: 692-697.

23 Pabst R, Tschernig T. Perivascular capillaries in the lung: an important but neglected vascular bed in immune reactions? J Allergy Clin Immunol 2002; 110: 209-214.

24 Uhlig S, Brasch F, Wollin L, Fehrenbach H, Richter J, Wendel A. Functional and fine structural changes in isolated rat lungs challenged with endotoxin ex vivo and in vitro. Am I Pathol 1995; 146: 1235-1247.

25 Parish WE, Akester AR, Gregg DM. The demonstration of bronchospasm in anaphylaxis by radiography. Int Arch Allergy Appl Immunol 1964; 25: 89-104.

26 Lai YL, Lamm WJ, Luchtel DL, Hildebrandt J. Massive postmortem bronchoconstriction in guinea pig lungs. $J$ Appl Physiol 1984; 56: 308-314.

27 Lai YL, Cornett AF. Substance P-inducing massive postmortem bronchoconstriction in guinea pig lungs. J Appl Physiol 1987; 62: 746-751.

28 Atzori L, Bannenberg G, Corriga AM, Moldeus P, Ryrfeldt A. Sulfur dioxide-induced bronchoconstriction in the isolated perfused and ventilated guinea-pig lung. Respiration 1992; 59: 16-21.

29 Gao Y, Raj JU. Role of veins in regulation of pulmonary circulation. Am J Physiol Lung Cell Mol Physiol 2005; 288: L213-L226.

30 Jonsson EW, Dahlèn SE. Interactions between leukotrienes and histamine in the anaphylactic contraction of guinea pig lung parenchyma. J Pharmacol Exp Ther 1994; 271: 615-623.

31 Bjorck T, Dahlèn SE. Leukotrienes and histamine mediate IgE-dependent contractions of human bronchi: pharmacological evidence obtained with tissues from asthmatic and non-asthmatic subjects. Pulm Pharmacol 1993; 6: 87-96.

32 Roquet A, Dahlen B, Kumlin M, et al. Combined antagonism of leukotrienes and histamine produces predominant inhibition of allergen-induced early and late phase airway obstruction in asthmatics. Am J Respir Crit Care Med 1997; 155: $1856-1863$.

33 Crosby JR, Cieslewicz G, Borchers M, et al. Early phase bronchoconstriction in the mouse requires allergen-specific IgG. J Immunol 2002; 168: 4050-4054.

34 Martin TJ, Broadley KJ. Mediators of adenosine- and ovalbumin-induced bronchoconstriction of sensitized guinea-pig isolated airways. Eur J Pharmacol 2002; 451: 89-99.

35 Rabe KF, Schmidt DT. Pharmacological treatment of asthma today. Eur Respir J 2001; 18: Suppl. 34, 34s-40s. 